\title{
Thermodynamic Partition Coefficients as a Versatile Alternative to Empirical Formula for Producing Gas Mixtures
}

\author{
Kai Fuchsberger \\ NMI Natural and Medical Sciences Institute at the University of Tübingen, Markwiesenstraße 55, 72770 Reutlingen, Germany
}

Received 3 September 2020; Accepted 26 February 2021

\begin{abstract}
When thinking about Henry's law one associates it with the solubility constant for gases in liquids at thermodynamic equilibrium. A practical application is checking gas sensors for specificity [1-3]. A gas sensor is for example the main part of an evidential breath analyser (EBA), a device to determine breath alcohol content of a person. Nowadays the empirical Dubowski formula, which is recommended officially, is solely suitable to convert aqueous ethanol concentration into ethanol concentration in air. Therefore, it is necessary to establish a theoretical based calculation method to enable synthesizing a humidified gas phase with any solute in a predefined concentration by dissolving the solute in water at the right concentration. This note will explain how to use Henry's law to derive an equation to perform this calculation for an arbitrtary solute. This equation, derived from Henry's law has the same predictive power compared to Dubowski formula.
\end{abstract}

Keywords: Gas sensor, evidential breath analyser, Dubowski equation, partition coefficient, selectivity

\section{Motivation}

Evaluation of evidential breath analysers (EBAs) uses artificial gas mixtures. These gases resemble exhaled air from a human at $34{ }^{\circ} \mathrm{C}$ and are composed of a water vapour saturated mixture of air and a compound of question. Calibration of an EBA generally requires many concentrations of ethanol. To test the response of EBAs to interfering substances [1-3] one requires gas mixtures with defined concentrations of an interfering compound [4]. A common way to produce a calibration gas is bubbling air through an aqueous ethanol solution with a specified concentration [5]. Dubowski's empirical formula [6] relates aqueous ethanol concentration $c_{a q}$ (ethanol) to the gas phase concentration $c_{g}$ (ethanol),

$k_{g / a q}($ ethanol $)=\frac{c_{g}(\text { ethanol })}{c_{a q}(\text { ethanol })}=4.145 \cdot 10^{-5} \cdot e^{0.06583 \cdot \frac{T}{{ }^{C} \mathrm{C}}}$.

Yet, no such equation is available to calculate these partition coefficients $k_{g / a}$ for other compounds. For this reason, here we derive a generic expression for the gas/liquid partition coefficient of an arbitrary substance.

\section{Derivation}

In thermodynamics for real solutions at low concentrations Henry's law Eq.2

$$
H^{c p}(x)=\frac{c_{a}(x)}{p(x)}
$$

relates aqueous concentrations $c_{a}$ of species $x$ to their partial pressure $p$ for a system in thermodynamic equilibrium $[7,8]$. For ambient conditions water-saturated air mixed with low amounts of a species $x$ is assumed to obey ideal gas law Eq.3 for an amount $n$ at a thermodynamic temperature $T$ in a volume $V$ with the gas constant $R=8.314 \mathrm{~J} \mathrm{~mol}^{-1} \mathrm{~K}^{-1}[7]$.

$p(x)=\frac{n(x)}{V} \cdot R \cdot T=c_{g}(x) \cdot R \cdot T$

Using Henry's constant at standard conditions $H$ with $T=298.15 \mathrm{~K}$ and its temperature dependency Eq.4

$\alpha(x)=\frac{d \ln (H)}{d \frac{1}{T}}$

for a species $x$ [8] results in an expression Eq.5 to convert aqueous concentrations $c_{a q}$ of an arbitrary species $x$ into gas phase concentration $c_{g}$.

$k_{g / a q}(x)=\frac{c_{g}(x)}{c_{a}(x)}=\frac{1}{H^{\Theta}(x) \cdot R \cdot T} \cdot e^{\alpha(x) \cdot\left(\frac{1}{T^{\Theta}}-\frac{l}{T}\right)}$

A detailed derivation of Eq. 5 is presented in the appendix.

\section{Comparison}

To check and compare the validity of Dubowski's formula and Eq. 5 temperature dependent partition coefficient $k_{g / a}$ data for ethanol in air/water system, extracted from figure 1 in [6], were used. Data extraction was done by using free online WebPlotDigitizer tool [9] and results are depicted in

Table 1 and Fig. 1. Calculating $\mathrm{R}^{2}$ with this data allows evaluation of the validity of both formulas. $\mathrm{R}^{2}$ is a measure of 
how well a model predicts measured values. The more $R^{2}$ approaches 1 , the better the predictive power of the model. For Dubowski's formula Eq. $1 R^{2}=0.996$ and for the Henry's law derived partition coefficient Eq. $5 R^{2}=0.997$. Obviously both models have the same high predictive power.

Table 1. Temperature dependent partition coefficients of ethanol in air ( $\mathrm{g}=$ gas) / water ( $\mathrm{a}=$ aqueous) system. (datapoints extracted from figure 1 in [6])

\begin{tabular}{c|c}
$T /{ }^{\circ} \mathrm{C}$ & $k_{g / a} / 10^{-5}$ \\
\hline 14.96 & 10.56 \\
14.96 & 10.70 \\
19.91 & 15.41 \\
19.94 & 15.85 \\
24.89 & 21.17 \\
24.95 & 21.61 \\
30.01 & 30.90 \\
29.98 & 31.87 \\
35.02 & 41.70 \\
35.02 & 42.08 \\
37.07 & 46.90 \\
37.10 & 48.00 \\
40.15 & 55.77 \\
40.18 & 56.78
\end{tabular}

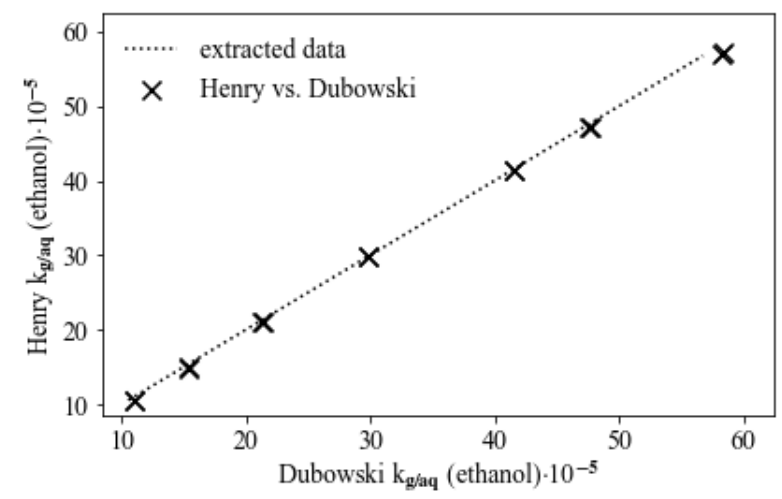

Fig. 1. Comparison of partition coefficients calculated with Dubowski's formula and Henry's law derived Eq.5 (using datapoints extracted from figure 1 belonging to reference [6]).

\section{Outlook}

This result allows to prepare humidified gases containing arbitrary compounds to e.g. check responses of EBAs [1-3]. This is because a simple setup, already established to calibrate EBAs with ethanol, can be used. This setup consists of a gaswashing bottle containing an aqueous solution of a compound with a known concentration $c_{a}$ in a temperature controlled environment, where air bubbles through. The concentration of the compound in the air passed through the gas-washing bottle can be calculated using Eq.5. A huge dataset containing constants for more than 4000 compounds is available and provided by Sander [8]. This allows to test gas sensors like EBAs in an environment as close as possible to the real world. Until now an alternative from [5] or [4] where dry air / compound mixture is suggested to be used, which in contrast to humidified air is far away from in vivo.

\section{Conclusion}

In this note a theoretical based calculation method based on thermodynamic laws was derived to calculate concentration of a compound in air in equilibrium with its aqueous solution. This kind of approach is superior to empirical ones (e.g. only available for the compound ethanol) due to physical meaningful constants and its general validity.

This is an Open Access article distributed under the terms of the Creative Commons Attribution License.

\section{References}

1. Dasgupta, A., Special concern. Accurate Results in the Clinical Laboratory, 459 (2019).

2. Adamski, J. and D. Zuba, Uncertainty of breath alcohol measurement. Problems of forensic sciences, 10139 (2015).

3. Jalal, A., Umasankar, Y., Gonzalez, P., Alfonso, A. and Bhansali, S., Multimodal technique to eliminate humidity interference for specific detection of ethanol. Biosensors and Bioelectronics, 87522 (2017).

4. OIML R 126 2012(E).

5. OIML R 126 1998(E).

\section{Appendix}

Derivation of Eq.5 starting with Eq.4:

$\alpha(x)=\frac{d \ln (H)}{d \frac{1}{T}}$

separation of variables $T$ and $H$

$d \ln (H)=\alpha(x) \cdot d \frac{1}{T}$
6. Dubowski, K., Breath-Alcohol Simulators: Scientific Basis and Actual Performance. Journal of Analytical Toxicology, 3(5) 177 (1997).

7. Atkins, P., Keeler, J. and De_Paula, J., Atkins' Physical Chemistry. Oxford: Oxford university press (2018).

8. Sander, R., Compilation of Henry's law constants (version 4.0) for water as solvent. Atmospheric Chemistry and Physics, 15(8) 4399 (2015).

9. https://automeris.io/WebPlotDigitizer/index.html

and integration

$\int_{H^{\theta}(x)}^{H(x, T)} d \ln (H)=\alpha(x) \cdot \int_{T^{\theta}}^{T} d \frac{1}{T}$

Yields

$[\ln (H)]_{H^{\theta}(x)}^{H(x, T)}=\alpha(x) \cdot\left[\frac{1}{T}\right]_{T^{\theta}}^{T}$ 
Or

$\ln \left(\frac{H(x, T)}{H^{\theta}(x)}\right)=\alpha(x) \cdot\left(\frac{1}{T}-\frac{1}{T^{\theta}}\right)$

solving for $H(x, T)$ and using Eq.2 yields

$H(x, T)=H^{\theta}(x) \cdot e^{\alpha(x) \cdot\left(\frac{1}{T} \cdot \frac{1}{T^{\theta}}\right)}=H^{c p}(x, T)=\frac{c_{a}(x, T)}{p(x, T)}$

followed transforming $p$ into $c_{g}$ using Eq.3 $\frac{c_{a}(x, T)}{c_{g}(x) \cdot R \cdot T}=H^{\theta}(x) \cdot e^{\alpha(x) \cdot\left(\frac{1}{T} \cdot \frac{1}{T^{\theta}}\right)}$

Taking the inverse on both sides

$\frac{c_{g}(x) \cdot R \cdot T}{c_{a}(x)}=\frac{1}{H^{\theta}(x)} \cdot e^{\alpha(x) \cdot\left(\frac{1}{T^{\prime}} \frac{1}{T^{\theta}}\right)}$

and solving for the partitioning coefficient $k_{g / a}$ to finally get Eq. 5

$k_{g / a}(x)=\frac{c_{g}(x)}{c_{a}(x)}=\frac{1}{H^{\Theta}(x) \cdot R \cdot T} \cdot e^{\alpha(x) \cdot\left(\frac{1}{T} \cdot \frac{1}{T^{\Theta}}\right)}$ 